\section{Randomized Trial on PET/CT Imaging Requires Adequate Follow-up}

TO THE EDITOR: With great interest did my colleagues and I read the article by Lebech et al. (1) on diagnostic imaging in patients with nonspecific symptoms or signs of cancer, and we commend the authors for using a randomized controlled design to compare PET/CT with CT imaging. However, the study appears to have examined a self-fulfilling prophecy, because PET/CT was evidently scrutinized less rigorously than CT.

Test accuracy was determined by comparing initial diagnostic test results (from either PET/CT or CT) with the final diagnosis, which was "based on a clinical approach using data obtained from all examinations." The acceptance of the final diagnosis as the reference standard may have led to bias, because patients received different additional diagnostic tests depending on the group they had been randomized to. On average, patients with suspected cancer after initial PET/CT underwent 1.4 tests, whereas patients in the CT group underwent 1.6 tests; however, the authors fail to present similar data on those patients in whom cancer was not suspected after initial imaging. Because of an overly optimistic trust in PET/CT, additional diagnostic measures might have been applied less frequently or less stringently in this group. In the worst scenario, a negative scan result was accepted as a truenegative result, without any further testing. A systematic followup after discharge would have been meaningful in order to verify that test-negative patients were truly free from disease.

In addition, randomized controlled trials on diagnostic imaging should examine patient-relevant outcomes rather than test accuracy measures, because the latter can also be analyzed in nonrandomized cohort studies, where each patient undergoes both imaging procedures. It is a promising finding that in the present trial, test accuracy was higher in the PET/CT group. But this finding is useful only if this advantage was not offset by false-negative results caused by an incomplete diagnostic workup. It would also have been important to examine whether the patients' fear of cancer decreased or their quality of life increased through better imaging. Furthermore, side effects caused directly by diagnostic testing or indirectly by inadequate treatment should have been recorded and reported. Finally, the study is not registered in a World Health Organization-certified study registry, even though study registration has been an established research standard for many years (2) — without a prespecified primary outcome measure, the results have to be regarded as explorative.

In conclusion, we recommend that the authors follow all patients for some months to make sure that the negative results observed were truly negative. Until further information is available, the authors' statement that "PET/CT is superior to CT" appears to be far too optimistic and requires confirmation by further, more robust trials.

\section{REFERENCES}

1. Lebech AM, Gaardsting A, Loft A, et al. Whole-body ${ }^{18} \mathrm{~F}-\mathrm{FDG}$ PET/CT is superior to CT as first-line diagnostic imaging in patients referred with serious nonspecific symptoms or signs of cancer: a randomized prospective study of 200 patients. J Nucl Med. 2017;58:1058-1064.

2. Deangelis CD, Drazen JM, Frizelle FA, et al. Is this clinical trial fully registered? A statement from the International Committee of Medical Journal Editors. JAMA. 2005;293:2927-2929.

\author{
Stefan Sauerland \\ Institute for Quality and Efficiency in Health Care \\ Im Mediapark 8 \\ 50670 Cologne, Germany \\ E-mail: stefan.sauerland@iqwig.de
}

Published online Mar. 2, 2017. DOI: 10.2967/jnumed.117.191346

\title{
Erratum
}

In the article "PSMA Ligands for PET Imaging of Prostate Cancer," by Schwarzenboeck et al. ( $\mathrm{J}$ Nucl Med. 2017;58:1545-1552), the last name of one of the authors was misspelled. "Ali Asfhar-Oromieh" should be "Ali Afshar-Oromieh." The authors regret the error. 